\title{
Prevalencia de tabaquismo y bajo desempeño escolar, en estudiantes de 11 a 24 años de edad del estado de Morelos, México
}

\author{
Edna A rillo-Santillán, ${ }^{1}$ Esteve Fernández, ${ }^{2}$ Mauricio Hernández-Avila, ${ }^{3}$ \\ Medardo Tapia-Uribe, ${ }^{4}$ A urelio Cruz-Valdés, ${ }^{3}$ Eduardo C Lazcano-Ponce. ${ }^{3}$
}

\begin{abstract}
Arillo-Santillán E, Fernández E, Hernández-Avila $M$, Tapia-Uribe M, Cruz-ValdésA, Lazcano-Ponce EC. Prevalencia de tabaquismo y bajo desempeño escolar, en estudiantes de 11 a 24 años de edad del estado de Morelos, México Salud Publica Mex 2002;44 supl 1:S54-S66. El texto completo en inglés de este artículo está disponible en: http://www.insp.mx/salud/index.html
\end{abstract}

\section{Resumen}

Objetivo. Estudiar la asociación entre desempeño escolar autorreportado, y la experimentación y consumo de tabaco actual en estudiantes adolescentes y adultos jóvenes del estado de Morelos, México. Material y métodos Se realizó un estudio poblacional transversal en el ámbito escolar, de 72 zonas administrativas de los 33 municipios del estado de Morelos, en áreas urbana, semiurbana y rural. De un marco muestral de escuelas públicas se seleccionaron aleatoriamente 13293 estudiantes, de entre 11 a 24 años, que respondieron un cuestionario autoaplicado. Se construyeron modelos de regresión logística multinomial, donde la variable dependiente fue el hábito tabáquico en tres categorías (no fumadores, fumadores experimentadores y fumadores actuales). Resultados La prevalencia de experimentación de consumo de tabaco fue $14.9 \%$ (IC 95\%: 14.1-15.7) en mujeres y $27.3 \%$ (IC 95\%: 26.1-28.4) en hombres; la prevalencia de consumo actual fue $6.1 \%$ (IC $95 \%$ : 5.6-6.6) y $13.1 \%$ (IC 95\%: 12.2-13.9), respectivamente. En ambos géneros, conforme se incrementa la prevalencia tanto en experimentación como en consumo de tabaco actual, decrece el desempeño escolar. La asociación entre consumo de tabaco actual
Arillo-Santillán E, Fernández E, Hernández-Avila M, Tapia-Uribe M, Cruz-Valdés A, Lazcano-Ponce EC. Smoking prevalence and low academic performance in students aged 11-24 years in Morelos, Mexico.

Salud Publica Mex 2002;44 suppl 1:S54-S66.

The English version of this paper is available at: http://www.insp.mx/salud/index.html

\begin{abstract}
A bstract
Objective To assess the association between self-reported academic performance and to bacco experimentation and established tobacco use among adolescents and young adults in Morelos State, Mexico. Material and Methods A population-based, cross-sectional study was carried out in 72 administrative areas from all 33 municipalities of Morelos State (urban, semi-urban and rural areas). A total of 13293 adolescents and yo ung adults aged 11 to 24 years were randomly selected from public schools. Data were collected using a self-administered questionnaire. Multinomial logistic regression models were fitted with smoking habit as the dependent variable with three categories (never a smoker, occasional smoker, and regular smokers). Results The prevalence of occasional smokers was $14.9 \%$ (95\% Cl: 14.115.7) among females and $27.3 \%$ (95\% Cl: 26.1-28.4) among males.The corresponding figures for females and males for established smokers were $6.1 \%(95 \% \mathrm{Cl}: 5.6-6.6)$ and $13.1 \%$ (95\% Cl: 12.2-13.9), respectively.A cademic performance selfreports were found to be inversely proportional to occasional or regular tobacco use. In women, the association with current tobacco consumption was 5.1 times higher
\end{abstract}

Este trabajo fue realizado con los fondos obtenidos de la Fundación Bristol Myers Squibb de N uevaYork, EUA, dentro de la convocatoria"Better health for women:A global health program". A simismo, el Consejo $\mathrm{N}$ acional de Ciencia y Tecnología de México, el Instituto N acional de Salud Pública de México y el Instituto C atalán de 0 ncología de Barcelona, España, financiaron parcialmente el análisis de la información.

(1) Secretaría Académica. Instituto N acional de Salud Pública. Cuernavaca, Morelos, México.

(2) Instituto C atalán de O ncología. Barcelona, España.

(3) Centro de Investigación en Salud Pública. Instituto N acional de Salud Pública. Cuernavaca, Morelos, México.

(4) Centro Regional de Investigaciones Multidisciplinarias. Universidad N acional Autónoma de México, Cuernavaca, Morelos, México.

Fecha de recibido: 30 marzo de 2001 • Fecha de aprobado: 5 de noviembre de 2001

Solicitud de sobretiros: Dr. Eduardo Lazcano Ponce. Dirección de Enfermedades Crónicas. Centro de Investigación en Salud Poblacional. Instituto N acional de Salud Pública. Avenida Universidad 655, Colonia Sta. Ma. A huacatitlán, 62508 C uernavaca, Morelos, México.

Correo electrónico: elazcano@ correo.insp.mx 
fue 5.1 veces mayor en mujeres (IC 95\%:2.5-10.4), con promedio anual de calificaciones de seis respecto de quienes reportaron un promedio con valores de 10 y de nueve; en hombres se observó el mismo fenómeno ( $\mathrm{RM}=4.2 ; \mathrm{IC}$ 95\%:2.7-6.7), con una tendencia lineal positiva y significativa en mujeres y hombres. Conclusiones Estos resultados brindan evidencia de la posible relación entre el incremento de consumo de tabaco y el bajo desempeño escolar.Dicha asociación está determinada por complejas prácticas cotidianas de estilos y condiciones de vida de los adolescentes. El texto completo en inglés de este artículo está disponible en: http://www.insp.mx/salud/index.html

Palabras clave: tabaquismo; rendimiento escolar bajo; adolescencia; estudiantes; México
(95\% Cl:2.5-10.4) in women with a yearly grade average of six or less, in comparison to those with scores of ten or nine. The same pattern was observed in men $(0 \mathrm{R}=4.2 ; 95 \%$ $\mathrm{Cl}: 2.7-6.7)$, with a significant linear trend both in both women and men. Conclusions. These results evidence that tobacco consumption is related to low academic performance. This relationships is determined by complex lifestyle patterns of adolescents. The English version of this paper is available at: http://www.insp.mx/salud/index.html

Key words: smoking; underachievement; adolescence; students; Mexico
$\mathrm{E}$ 1 consumo de tabaco constituye la primera causa de pérdida de la salud, así como la primera causa de muerte prematura y evitable en países desarrollados. ${ }^{1}$ En este contexto, cerca de $20 \%$ de los 7 a 10 millones de muertes que se presentaron en el año 2000 podrían haberse prevenido, si el consumo de tabaco se hubiera eliminado hace 15 años. ${ }^{2}$ En México, en los últimos 10 años se ha incrementado el número de fumadores de 9 a 13 millones. ${ }^{3}$ La Encuesta Nacional de Adicciones (ENA) 1998, que incluye población residente de zonas urbanas, con edades entre 12 y 65 años, reportó una prevalencia de $16.3 \%$ en mujeres y de $42.9 \%$ en hombres. La mayoría de estos fumadores tuvieron entre 18 y 29 años. La proporción de fumadores que iniciaron el consumo de tabaco antes de los 18 años muestra una tendencia ascendente, según los datos de las diferentes encuestas (52.2\% en 1988, $56.8 \%$ en 1993 y 61.4\% en 1998). También la prevalencia de tabaquismo entre menores de 17 años aumentó, de $7.7 \%$ en $1988^{4}$ a $11.6 \%$ en $1998 .^{3}$ Asimismo, la prevalencia de consumo de tabaco alguna vez en la vida de los adolescentes ha sido reportada en $42.2 \%$, de acuerdo con la Encuesta Nacional sobre el Uso de Drogas entre la Comunidad Escolar de México. ${ }^{5}$

Con estos antecedentes, posiblemente México se encuentre en la segunda de las cuatro etapas que caracterizan el modelo descriptivo de la epidemia del tabaco. ${ }^{6}$ En ésta se aprecia una mayor prevalencia de tabaquismo en hombres que en mujeres, una prevalencia baja de ex fumadores, y una prevalencia mayor de consumo de tabaco en las clases altas. En esta etapa los riesgos de exposición al tabaco no están ampliamente entendidos, pues las tasas de mortalidad, atribuidas al tabaquismo, son relativamente bajas porque el impacto en la salud se constatará dos o tres décadas más tarde. En México, se estima que en los próximos años se presentarán unas 10000 muertes anuales por cáncer de pulmón. ${ }^{7}$

Asimismo, en México no han sido suficientemente difundidas las implicaciones que el tabaco como factor de riesgo tiene en la salud reproductiva. ${ }^{8}$ Además, se han desarrollado escasos estudios de exposición al humo del cigarrillo con una perspectiva de género. ${ }^{9}$

Existen diversos estudios que muestran la asociación del hábito tabáquico en los adolescentes, con una gran variedad de factores tanto sociales, económicos, ambientales, biológicos y personales. ${ }^{10}$ Uno de ellos es el desempeño escolar, indicador que refleja características de compromiso educacional, motivación, competencia y éxito escolar. ${ }^{11,12}$ Algunos trabajos refieren una mayor prevalencia de consumo de tabaco, asociada con la autopercepción de un pobre desempeño académico. ${ }^{11-13}$ Así también, en diversas poblaciones de adolescentes, se ha evaluado el bajo desempeño escolar -medido de diversas manerasasociado con mayor consumo de tabaco y otros factores. $^{14-19}$

Sin embargo, en la población mexicana no existe un estudio que haya caracterizado la asociación entre desempeño escolar y consumo de tabaco, que constituye una expresión de determinados patrones de estilo de vida. Por ello, el objetivo de este trabajo es cuantificar diferencialmente la asociación entre desempeño escolar y prevalencia de experimentación y consumo actual de tabaco en estudiantes de Morelos, México.

\section{Material y métodos}

La información del presente estudio proviene de una investigación multidisciplinaria e interinstitucional realizada por el Instituto Nacional de Salud Pú- 
blica (INSP), durante el ciclo escolar 1998-1999, en el estado de Morelos, en México, en colaboración con las Secretarías de Salud y Educación Pública locales, instituciones educativas y organizaciones no gubernamentales. Se trata de la primera fase (medición basal) de un estudio de cohorte a gran escala en estudiantes de Morelos. Se realizó una encuesta transversal en el ámbito escolar, con representación estatal.

Se desarrolló un marco muestral de escuelas públicas de nivel medio (secundarias), medio superior (preparatorias) y superior (licenciaturas universitarias); por lo que la unidad de muestreo fueron las escuelas, todas ellas con la misma probabilidad de selección. El marco fue conformado por 260 secundarias, con una población escolar de 60686 alumnos; 92 preparatorias, con 18067 alumnos; y una universidad pública con 15 escuelas, con 7452 estudiantes. Todas corresponden a las 72 zonas administrativas de los 33 municipios del estado de Morelos, pertenecientes a las áreas urbana, semiurbana y rural. De este marco se seleccionaron aleatoriamente 13293 adolescentes y adultos jóvenes, hombres y mujeres de entre 11 a 24 años de edad.

Los estudiantes seleccionados, luego de un consentimiento informado, respondieron en la escuela un cuestionario autoaplicado. La tasa de respuesta obtenida fue de $98.6 \%$. La encuesta incluye varias secciones y las consideradas en este estudio fueron: a) características sociodemográficas (edad, ingreso familiar, lugar de residencia, ocupación de los padres, condiciones de la vivienda, otras); b) desempeño escolar, a partir del promedio de calificaciones durante el último año en escala de 1 a 10;c) antecedente de adicciones de los padres (tabaquismo, alcoholismo y drogas ilegales), y d) adicciones en los estudiantes (tabaquismo, alcoholismo y drogas ilegales).

Se definió el tabaquismo actual como el consumo de aquellos individuos que en el momento de la encuesta refirieron consumir al menos un cigarrillo al día. Como experimentadores, aquellos sujetos que reportaron haber fumado previamente y que en el momento actual han dejado de hacerlo, o aquellos que autorreportaron dar sólo algunas fumadas actualmente. Y como no fumadores, a quienes reportaron no haber fumado ni fumar cigarrillos. Estas categorías reflejan el proceso de inicio del tabaquismo establecido por Flay; ${ }^{20}$ debido a los datos disponibles, se han considerado conjuntamente los adolescentes que prueban y aquellos que experimentan.

El desempeño, para fines de este trabajo, se define como el nivel de éxito alcanzado en alguna área específica, en este caso en la escolar. Se construyó un índice considerando el autorreporte del estudiante de la calificación promedio que obtuvo el año inmediato anterior (escala de 0 a 10); se categorizó en cuatro estratos: 10 o nueve (alto), ocho (medio), siete (bajo), e igual o menor de seis (muy bajo). Para algunos casos, por criterio estadístico, se unieron los estratos bajo y muy bajo.

El tabaquismo en los padres se definió a partir del reporte de los estudiantes sobre su percepción del consumo de tabaco de éstos.

El lugar de residencia fue establecido de acuerdo con la categorización municipal que ha sido desarrollada por el Instituto Nacional de Estadística, Geografía e Informática (INEGI), con base en el Censo Nacional de Población y Vivienda, que utiliza como indicadores el número de habitantes y nivel de marginación.

Para el índice de nivel socioeconómico se generó una variable ordinal del 1 al 10, mediante un análisis de componentes principales. Las variables utilizadas fueron características de la vivienda (tipo de piso, presencia de agua potable y drenaje), ingreso familiar, así como disponibilidad de casa propia, auto, gas estacionario, refrigerador, teléfono, televisión a color y videocasetera. El índice se conformó en tres categorías (bajo, medio y alto), que se agruparon a partir de los percentiles.

La proporción de sujetos no fumadores, experimentadores y fumadores actuales, de acuerdo con los estratos de desempeño escolar, se analizó separadamente para hombres y mujeres, considerando las diferencias de género existentes en el consumo de tabaco. ${ }^{20-22}$ Además, se realizó un análisis estratificado por las siguientes variables: edad (11-12, 13-14, 15-17 y 18-24 años); índice de nivel socioeconómico (bajo, medio y alto); lugar de residencia (rural, semiurbana y urbana); intoxicación aguda por alcohol al menos una vez al mes (no, sí); consumo de alguna droga ilegal en el pasado o actual (no, sí); así como antecedente de consumo de tabaco en el padre (no, sí), y en la madre (no, sí).

Se construyeron modelos logísticos multinominales, ${ }^{23}$ donde la variable dependiente fue el hábito tabáquico en tres categorías (no fumadores, fumadores experimentadores y fumadores establecidos, considerando como categoría de referencia a los no fumadores). Estos modelos proporcionaron la razón de momios $(R M)$ y su correspondiente intervalo de confianza al 95\% (IC). Los modelos fueron realizados en cada categoría de exposición y evalúan el efecto independiente de desempeño escolar por cada una de las variables de estudio, estimando, asimismo, la tendencia o el gradiente dosis respuesta de la posibilidad de fumar en función del desempeño escolar. El análisis se hizo utilizando el paquete estadístico STATA.

El gradiente dosis respuesta se obtuvo mediante la prueba de tendencia lineal de Mantel-Haenszel, 
calculada mediante la diferencia de lejanías o deviances, entre el modelo con la variable desempeño escolar en escala ordinal, y el modelo sin esta variable.

\section{Resultados}

La delimitación de la muestra estudiada, según las principales variables sociodemográficas, el consumo de tabaco y otras variables relacionadas, se muestran en el cuadro I. La prevalencia de experimentación de consumo de tabaco fue $14.9 \%$ (IC 95\%: 14.1-15.7) en mujeres, y 27.3\% (IC 95\%: 26.1-28.4) en hombres; asimismo, la prevalencia de consumo de tabaco actual fue 6.1\% (IC 95\%: 5.6-6.6) y 13.1\% (IC 95\%: 12.2-13.9), respectivamente.

Conviene destacar que, en cuanto al desempeño escolar, la proporción de mujeres en el estrato considerado alto, es decir, con promedio anual de valores de $10 \mathrm{y}$ de nueve, es mayor (32.7\%) que el de los hombres $(20.9 \%)$; esta relación se invierte en el estrato bajo, de promedio anual igual a siete (17.1\% mujeres y $26.4 \%$ hombres).

En los cuadros II y IV se muestra la delimitación de las principales variables, según el hábito tabáquico y el desempeño escolar, tanto para mujeres como para hombres.

En las mujeres, como se observa en el cuadro III la prevalencia de experimentación de tabaco es mayor entre las que reportan un muy bajo desempeño esco$\operatorname{lar}(R M=4.1$, IC 95\%:2.6-6.5, en aquellas con desempeño igual o menor de 6 , respecto de las que tienen un promedio de 10 o de nueve), con una tendencia lineal estadísticamente significativa (figura 1). En lo que se refiere al consumo actual, la posibilidad de ser fumadora fue cinco veces mayor entre las que tenían desempeño bajo, tras ajustar por el resto de variables, con un patrón similar al descrito para las experimentadoras.

En los hombres, se puede observar en el modelo total (cuadro V), que, tanto en experimentadores como en consumidores actuales, existió una mayor asociación de consumo de tabaco en los estudiantes, con un promedio igual o menor a seis. Asimismo, existió una asociación inversa entre consumo de tabaco actual y desempeño escolar, pues la posibilidad de ser fumador fue 4.2 veces mayor entre los estudiantes con desempeño escolar igual o menor de seis, con una tendencia lineal estadísticamente significativa (figura 2).

La asociación entre desempeño escolar y hábito tabáquico en mujeres se examina en los diferentes estratos del resto de las variables (cuadro III). Cabe destacar la consistencia del patrón, tanto para experimentadoras como para fumadoras actuales, más acen-

\section{Cuadro I \\ Características de los 13293 estudiantes del estudio de Morelos, México, 1998-1999}

\begin{tabular}{|c|c|c|}
\hline $\begin{array}{c}\text { Mujeres } \\
\text { (7 } 468-56.2 \%)\end{array}$ & $\begin{array}{c}\text { Hombres } \\
\text { (5 } 825-43.8 \%)\end{array}$ & $\begin{array}{c}\text { Total } \\
\text { (13 293) }\end{array}$ \\
\hline Número \% & Número $\%$ & Número $\%$ \\
\hline
\end{tabular}

Edad (en años)

\begin{tabular}{rrrrrrr}
$11-12$ & 1957 & 26.2 & 1694 & 29.1 & 3651 & 27.5 \\
\hline $13-14$ & 3374 & 45.2 & 2748 & 47.2 & 6122 & 46.0 \\
\hline $15-17$ & 1430 & 19.1 & 811 & 13.9 & 2241 & 16.9 \\
\hline $18-24$ & 707 & 9.5 & 572 & 9.8 & 1279 & 9.6
\end{tabular}

Indice de nivel socioeconómico

\begin{tabular}{lllllll} 
Bajo & 2218 & 29.7 & 1114 & 19.1 & 3332 & 25.1 \\
\hline Medio & 3399 & 45.5 & 3440 & 59.1 & 6839 & 51.4 \\
\hline Alto & 1851 & 24.8 & 1271 & 21.8 & 3122 & 23.5
\end{tabular}

Lugar de residencia

\begin{tabular}{lllllll} 
Rural & 2863 & 38.4 & 2406 & 41.3 & 5269 & 39.7 \\
\hline Semi-urbana & 1959 & 26.2 & 1301 & 22.3 & 3260 & 24.5 \\
\hline Urbana & 2446 & 35.4 & 2118 & 36.4 & 4764 & 35.8
\end{tabular}

Desempeño escolar

\begin{tabular}{lrrrrrr}
$10-9$ & 2443 & 32.7 & 1215 & 20.9 & 3658 & 27.5 \\
\hline 8 & 3642 & 48.8 & 2874 & 49.3 & 6516 & 49.0 \\
\hline 7 & 1278 & 17.1 & 1537 & 26.4 & 2815 & 21.2 \\
\hline$\leq 6$ & 105 & 1.4 & 194 & 3.4 & 304 & 2.3
\end{tabular}

Consumo de tabaco

\begin{tabular}{lrrrrrr} 
No fumador & 5899 & 79.0 & 3474 & 59.6 & 9373 & 70.5 \\
\hline Experimentador & 1114 & 14.9 & 1590 & 27.3 & 2704 & 20.3 \\
\hline Fumador actual & 455 & 6.1 & 761 & 13.1 & 1216 & 9.2
\end{tabular}

Intoxicación aguda por alcohol

\begin{tabular}{lrrrrrr} 
No & 7026 & 94.1 & 5452 & 93.6 & 12478 & 93.9 \\
\hline Sí & 442 & 5.9 & 373 & 6.4 & 815 & 6.1
\end{tabular}

Consumo de alguna droga ilegal

\begin{tabular}{lrrrrrr} 
No & 7221 & 96.7 & 5298 & 90.9 & 12519 & 94.2 \\
\hline Sí & 247 & 3.3 & 527 & 9.1 & 774 & 5.8
\end{tabular}

Hábito tabáquico del padre

\begin{tabular}{lrrrrrr} 
No & 7169 & 96.0 & 4782 & 82.1 & 11951 & 89.9 \\
\hline Sí & 299 & 4.0 & 1043 & 17.9 & 1342 & 10.1
\end{tabular}

Hábito tabáquico de la madre

\begin{tabular}{lrrrrrr} 
No & 7325 & 98.1 & 5502 & 94.4 & 12827 & 96.5 \\
\hline Sí & 143 & 1.9 & 323 & 5.6 & 466 & 3.5
\end{tabular}




\section{Cuadro II \\ Distribución de Las estudiantes según el deSempeño escolar, CONSUMO de tabaco, DIFERENTES VARIABLES SOCIOECONÓMICAS Y ANTECEDENTES PERSONALES Y FAMILIARES. \\ Morelos, México, 1998-1999}

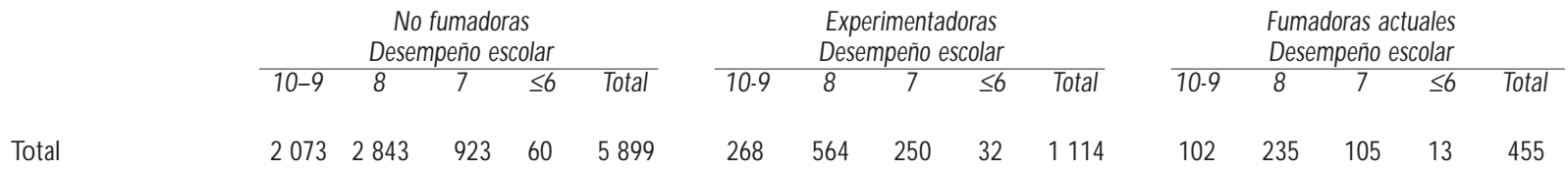

Edad (en años)

\begin{tabular}{rrrrrrrrrrrrrrrr}
$11-12$ & 622 & 782 & 226 & 18 & 1648 & 62 & 102 & 40 & 6 & 210 & 21 & 55 & 19 & 4 & 99 \\
\hline $13-14$ & 935 & 1331 & 451 & 25 & 2742 & 105 & 268 & 103 & 14 & 490 & 36 & 68 & 35 & 3 & 142 \\
\hline $15-17$ & 374 & 499 & 160 & 14 & 1047 & 67 & 138 & 70 & 8 & 283 & 22 & 48 & 27 & 3 & 100 \\
\hline $18-24$ & 142 & 231 & 86 & 3 & 462 & 34 & 56 & 37 & 4 & 131 & 23 & 64 & 24 & 3 & 114
\end{tabular}

Indice de nivel socioeconómico

\begin{tabular}{|c|c|c|c|c|c|c|c|c|c|c|c|c|c|c|c|}
\hline Bajo & 549 & 964 & 331 & 22 & 1866 & 60 & 147 & 59 & 9 & 275 & 12 & 33 & 29 & 3 & 77 \\
\hline Medio & 929 & 1332 & 449 & 30 & 2740 & 120 & 246 & 131 & 17 & 514 & 30 & 78 & 35 & 2 & 145 \\
\hline Alto & 595 & 547 & 143 & 8 & 1293 & 88 & 171 & 60 & 6 & 325 & 60 & 124 & 41 & 8 & 233 \\
\hline
\end{tabular}

Lugar de residencia

\begin{tabular}{|c|c|c|c|c|c|c|c|c|c|c|c|c|c|c|c|}
\hline Rural & 816 & 1195 & 389 & 29 & 2429 & 88 & 169 & 93 & 11 & 361 & 16 & 33 & 19 & 5 & 73 \\
\hline Semiurbana & 552 & 729 & 266 & 16 & 1563 & 86 & 139 & 78 & 15 & 318 & 11 & 40 & 25 & 2 & 78 \\
\hline Urbana & 705 & 919 & 268 & 15 & 1907 & 94 & 256 & 79 & 6 & 435 & 75 & 162 & 61 & 6 & 304 \\
\hline
\end{tabular}

Intoxicación aguda por alcohol

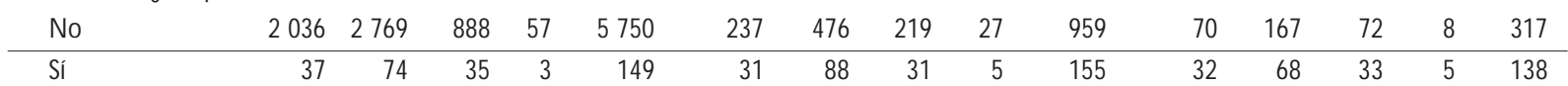

Consumo de alguna droga ilegal

\begin{tabular}{|c|c|c|c|c|c|c|c|c|c|c|c|c|c|c|c|}
\hline No & 2046 & 2801 & 909 & 60 & 5816 & 254 & 509 & 230 & 29 & 1022 & 87 & 200 & 84 & 12 & 383 \\
\hline Sí & 27 & 42 & 14 & 0 & 83 & 14 & 55 & 20 & 3 & 92 & 15 & 35 & 21 & 1 & 77 \\
\hline
\end{tabular}

Hábito tabáquico del padre

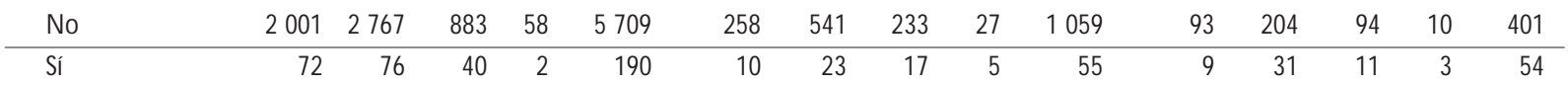

Hábito tabáquico de la madre

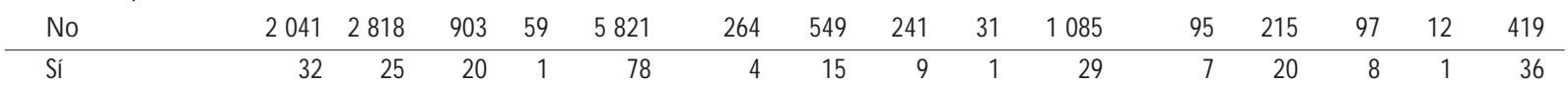

tuado en las jóvenes de 18 a 24 años, y en aquéllas con mayor nivel socioeconómico y con residencia semiurbana y rural. No se logró evidenciar un efecto entre bajo desempeño escolar y consumo de tabaco en mujeres expuestas a intoxicación aguda por alcohol, aunque se destaca que la prevalencia de intoxicación fue baja. La asociación independiente entre intoxicación aguda por alcohol y consumo de tabaco fue importante, considerando el resto de los valores del modelo total ( $R M=3.4$; IC 95\%:2.6-4.7 en las experimentadoras y $R M=8.0$; IC 95\%:5.8-10.9 en las fumadoras establecidas; datos no mostrados en cuadro). En el caso de quienes reportaron consumir alguna droga en el pasado o actualmente, la asociación entre desempeño y consumo de tabaco fue más marcada. Respecto del antecedente de tener padre o madre fumadora, 


\section{Cuadro III \\ Asociación* entre el desempeño escolar y CONSUMO de tABaco en LAS ESTUdiantes, SEGÚN DIFERENTES VARIABLES SOCIECONÓMICAS, ANTECEDENTES PERSONALES Y FAMILIARES. Morelos, MÉXICO, 1998-1999}

\begin{tabular}{|c|c|c|c|c|c|c|c|c|c|}
\hline \multirow[b]{2}{*}{$\begin{array}{l}\text { Desempeño } \\
\text { escolar }\end{array}$} & \multirow[b]{2}{*}{$10-9^{\ddagger}$} & \multicolumn{4}{|c|}{ Experimentadoras } & \multicolumn{4}{|c|}{ Fumadoras actuales } \\
\hline & & $\begin{array}{c}8 \\
\text { RM IC } 95 \%\end{array}$ & $\begin{array}{c}7 \\
\text { RM IC } 95 \%\end{array}$ & $\begin{aligned} & \leq 6 \\
& \text { RM IC } 95 \%\end{aligned}$ & $\begin{array}{l}\chi^{2} \text { tendencia } \\
\text { (p) }\end{array}$ & $\begin{array}{c}8 \\
\text { RM IC } 95 \%\end{array}$ & $\begin{array}{c}7 \\
\text { RM IC } 95 \%\end{array}$ & $\begin{array}{cl} & \leq 6 \\
\text { RM IC } 95 \%\end{array}$ & $\begin{array}{l}\chi^{2} \text { tendencia } \\
\text { (p) }\end{array}$ \\
\hline Modelo total & 1.0 & $1.5(1.3-1.8)$ & $2.1(1.7-2.5)$ & $4.1(2.6-6.5)$ & $70.0(p<0.001)$ & $1.7(1.3-2.2)$ & $2.5(1.8-3.5)$ & $5.1(2.5-10.4)$ & $39.4(p<0.001)$ \\
\hline \multicolumn{10}{|l|}{ Edad (en años) } \\
\hline $11-12$ & 1.0 & $1.3(1.0-1.9)$ & $1.9(1.2-3.0)$ & $3.1(1.2-8.5)$ & $10.0(p<0.01)$ & $2.3(1.3-3.9)$ & $3.9(1.9-7.7)$ & $10.1(2.8-36.1)$ & $23.4(p<0.001)$ \\
\hline $13-14$ & 1.0 & $1.8(1.4-2.3)$ & $2.0(1.2-2.8)$ & $4.5(2.1-9.2)$ & $29.8(p<0.001)$ & $1.4(0.9-2.2)$ & $2.4(1.4-4.1)$ & $3.1(0.7-12.4)$ & $9.6(p<0.01)$ \\
\hline $15-17$ & 1.0 & $1.3(0.7-1.6)$ & $2.4(1.3-4.6)$ & $4.7(1.2-18.9)$ & $22.4(p<0.001)$ & $1.3(0.7-2.2)$ & $2.4(1.3-4.6)$ & $4.7(1.2-18.9)$ & $9.2(p<0.01)$ \\
\hline $18-24$ & 1.0 & $1.0(0.6-1.6)$ & $1.7(0.9-3.0)$ & $6.1(1.2-31.1)$ & $5.4(p<0.05)$ & $1.9(1.1-3.4)$ & $1.8(0.9-3.7)$ & $6.2(0.9-42.7)$ & $2.6(p=0.11)$ \\
\hline
\end{tabular}

Indice de nivel socioeconómico

\begin{tabular}{lllllllllc} 
Bajo & 1.0 & $1.3(1.0-1.8)$ & $1.5(1.0-2.3)$ & $3.7(1.6-8.5)$ & $8.4(p<0.01)$ & $1.5(0.7-3.0)$ & $3.7(1.8-7.7)$ & $6.3(1.6-25.3)$ & $16.4(p<0.001)$ \\
\hline Medio & 1.0 & $1.4(1.1-1.7)$ & $2.0(1.5-2.7)$ & $3.4(1.7-6.5)$ & $31.0(p<0.001)$ & $1.6(1.0-2.5)$ & $2.0(1.1-3.3)$ & $1.4(0.3-6.6)$ & $5.4(p<0.05)$ \\
\hline Alto & 1.0 & $2.0(1.5-2.6)$ & $2.7(1.8-3.9)$ & $5.6(1.8-17.2)$ & $35.0(p<0.001)$ & $2.0(1.4-2.9)$ & $2.6(1.6-4.2)$ & $14.1(4.2-47.6)$ & $21.4(p<0.001)$
\end{tabular}

Lugar de residencia

\begin{tabular}{lccccccccc} 
Rural & 1.0 & $1.3(1.0-1.7)$ & $2.2(1.6-3.0)$ & $3.1(1.4-6.6)$ & $24.8(p<0.001)$ & $1.1(0.6-2.1)$ & $2.0(1.0-4.2)$ & $5.3(1.6-17.7)$ & $7.6(p<0.01)$ \\
\hline Semiurbana & 1.0 & $1.2(0.9-1.7)$ & $1.7(1.2-2.4)$ & $5.5(2.5-11.8)$ & $17.6(p<0.001)$ & $2.6(1.3-5.3)$ & $3.7(1.7-8.0)$ & $5.7(1.1-30.0)$ & $12.6(p<0.001)$ \\
& & & & & & & & & \\
Urbana & 1.0 & $2.1(1.6-2.7)$ & $2.2(1.6-3.1)$ & $2.9(1.1-8.1)$ & $25.6(p<0.001)$ & $1.8(1.3-2.4)$ & $2.3(1.6-3.5)$ & $3.8(1.2-11.7)$ & $19.2(p<0.001)$
\end{tabular}

Intoxicación aguda por alcohol

\begin{tabular}{cccccccc} 
No & 1.0 & $1.5(1.3-1.8)$ & $2.3(1.9-2.8)^{\S}$ & $66.6(p<0.001)$ & $1.9(1.4-2.5)$ & $2.8(2.0-4.0)^{\S}$ & $36.2(p<0.001)$ \\
\hline Sí & 1.0 & $1.4(0.8-2.5)$ & $1.2(0.6-2.4)^{\S}$ & $0.2(p=0.65)$ & $1.1(0.6-2.0)$ & $1.6(0.8-3.3)^{\S}$ & $1.8(p=0.18)$
\end{tabular}

Consumo de alguna droga

\begin{tabular}{llllrrrr} 
No & 1.0 & $1.5(1.3-1.8)$ & $2.2(1.8-2.6)^{5}$ & $59.4(p<0.001)$ & $1.7(1.3-2.3)$ & $2.6(1.9-3.6)^{5}$ & $31.0(p<0.001)$ \\
\hline Sí & 1.0 & $2.7(1.2-5.9)$ & $3.5(1.3-9.3)^{5}$ & $7.4(p<0.01)$ & $1.8(0.7-4.6)$ & $3.5(1.2-10.3)^{\S}$ & $5.8(p<0.05)$
\end{tabular}

Hábito tabáquico del padre

\begin{tabular}{lllllllr} 
No & 1.0 & $1.5(1.3-1.8)$ & $2.1(1.7-2.6)^{5}$ & $57.2(p<0.001)$ & $1.6(1.2-2.1)$ & $2.7(1.9-3.7)^{5}$ & $33.2(p<0.001)$ \\
\hline Sí & 1.0 & $2.3(1.0-5.4)$ & $2.8(1.1-7.0)^{5}$ & $5.2(p<0.05)$ & $3.6(1.5-8.8)$ & $2.7(0.9-7.8)^{5}$ & $4.0(p<0.05)$
\end{tabular}

Hábito tabáquico de la madre

\begin{tabular}{lccccccc} 
No & 1.0 & $1.5(1.3-1.7)$ & $2.1(1.8-2.6)^{5}$ & $59.2(p<0.001)$ & $1.6(1.2-2.1)$ & $2.7(2.0-3.7)^{\S}$ & $34.4(p<0.001)$ \\
\hline Sí & 1.0 & $5.9(1.5-22.8)$ & $5.3(1.2-22.7)^{5}$ & $3.8(p=0.051)$ & $5.1(1.5-16.9)$ & $2.4(0.6-9.4)^{\S}$ & $1.8(p=0.18)$
\end{tabular}

\footnotetext{
* RM ajustada por edad, nivel socioeconómico, lugar de residencia, intoxicación aguda por consumo de alcohol al menos una vez al mes, consumo pasado 0 actual de alguna droga ilegal, hábito tabáquico del padre y de la madre

₹ Categoría de referencia

$\S$ RM para puntuación en el desempeño escolar $\leq 7$
}

la asociación es evidente en las experimentadoras $(R M=2.8$; IC 95\%: 1.1-7.0, para antecedente en el padre; y $R M=5.3$; IC 95\%:1.2-22.7, para antecedente en la madre).
En los hombres (cuadro V), tanto la experimentación como el consumo de tabaco actual presentan un efecto dosis respuesta en todos los grupos. Los estimadores de efecto son más altos en aquellos con ín- 


\section{Cuadro IV \\ Distribución de los estudiantes Según el DeSempeño escolar, CONSUMO de tABaco, DIFERENTES VARIABLES SOCIOECONÓMICAS Y ANTECEDENTES PERSONALES Y FAMILIARES. \\ Morelos, México, 1998-1999}

\begin{tabular}{|c|c|c|c|c|c|c|c|c|c|c|c|c|c|c|}
\hline & & $\begin{array}{r}N \\
\text { Dese }\end{array}$ & $\begin{array}{l}\text { umado } \\
\text { beño }\end{array}$ & & & & & $\begin{array}{l}\text { imen } \\
\text { peño }\end{array}$ & & & & & pe & \\
\hline & $10-9$ & 8 & 7 & $\leq 6$ & Total & $10-9$ & 8 & 7 & $\leq 6$ & Total & $10-9$ & 8 & . & Total \\
\hline & 888 & 1668 & 823 & 95 & 3474 & 238 & 832 & 459 & 61 & 1590 & 89 & 374 & & 761 \\
\hline
\end{tabular}

Edad (en años)

\begin{tabular}{rrrrrrrrrrrrrrrr}
$11-12$ & 304 & 521 & 248 & 26 & 1099 & 87 & 204 & 94 & 11 & 396 & 29 & 96 & 62 & 12 & 199 \\
\hline $13-14$ & 407 & 845 & 400 & 45 & 1697 & 101 & 410 & 242 & 34 & 787 & 29 & 139 & 82 & 14 & 264 \\
\hline $15-17$ & 108 & 187 & 102 & 17 & 414 & 31 & 138 & 75 & 11 & 255 & 14 & 62 & 58 & 8 & 142 \\
\hline $18-24$ & 69 & 115 & 73 & 7 & 264 & 19 & 80 & 48 & 5 & 152 & 17 & 77 & 53 & 9 & 156
\end{tabular}

\begin{tabular}{|c|c|c|c|c|c|c|c|c|c|c|c|c|c|c|c|}
\hline Indice de $n$ & & & & & & & & & & & & & & & \\
\hline Bajo & 146 & 312 & 182 & 26 & 666 & 47 & 178 & 83 & 13 & 321 & 12 & 67 & 41 & 7 & 127 \\
\hline Medio & 520 & 1051 & 502 & 57 & 2130 & 144 & 504 & 294 & 43 & 985 & 31 & 156 & 112 & 26 & 325 \\
\hline Alto & 222 & 305 & 139 & 12 & 678 & 47 & 150 & 82 & 5 & 284 & 46 & 151 & 102 & 10 & 309 \\
\hline
\end{tabular}

\begin{tabular}{|c|c|c|c|c|c|c|c|c|c|c|c|c|c|c|c|}
\hline \multicolumn{16}{|l|}{ Lugar de residencia } \\
\hline Rural & 373 & 766 & 360 & 48 & 1547 & 100 & 354 & 204 & 29 & 687 & 17 & 82 & 56 & 17 & 172 \\
\hline Semiurbana & 209 & 357 & 201 & 20 & 787 & 63 & 185 & 109 & 14 & 371 & 13 & 77 & 45 & 8 & 143 \\
\hline Urbana & 306 & 545 & 262 & 27 & 1140 & 75 & 293 & 146 & 18 & 532 & 59 & 215 & 154 & 18 & 446 \\
\hline
\end{tabular}

Intoxicación aguda por alcohol

\begin{tabular}{|c|c|c|c|c|c|c|c|c|c|c|c|c|c|c|c|}
\hline No & 877 & 1636 & 798 & 92 & 3403 & 227 & 758 & 416 & 56 & 1457 & 77 & 294 & 188 & 33 & 592 \\
\hline Sí & 11 & 32 & 25 & 3 & 71 & 11 & 74 & 43 & 5 & 133 & 12 & 80 & 67 & 10 & 169 \\
\hline
\end{tabular}

Consumo de alguna droga ilegal

\begin{tabular}{|c|c|c|c|c|c|c|c|c|c|c|c|c|c|c|c|}
\hline No & 861 & 1621 & 795 & 90 & 3367 & 217 & 734 & 394 & 52 & 1397 & 69 & 269 & 168 & 28 & 534 \\
\hline Sí & 27 & 47 & 28 & 5 & 107 & 21 & 98 & 65 & 9 & 193 & 20 & 105 & 87 & 15 & 227 \\
\hline
\end{tabular}

\begin{tabular}{|c|c|c|c|c|c|c|c|c|c|c|c|c|c|c|c|}
\hline \multicolumn{16}{|c|}{ Hábito tabáquico del padre } \\
\hline No & 754 & 1401 & 698 & 79 & 2932 & 188 & 665 & 381 & 45 & 1279 & 60 & 288 & 190 & 33 & 571 \\
\hline Sí & 134 & 267 & 125 & 16 & 542 & 50 & 167 & 78 & 16 & 311 & 29 & 86 & 65 & 10 & 190 \\
\hline
\end{tabular}

Hábito tabáquico de la madre

\begin{tabular}{|c|c|c|c|c|c|c|c|c|c|c|c|c|c|c|c|}
\hline No & 846 & 1602 & 777 & 91 & 3316 & 223 & 792 & 442 & 57 & 1514 & 76 & 329 & 227 & 40 & 672 \\
\hline Sí & 42 & 66 & 46 & 4 & 158 & 15 & 40 & 17 & 4 & 76 & 13 & 45 & 28 & 3 & 89 \\
\hline
\end{tabular}

dice de nivel socioeconómico medio, y lugar de residencia urbana, en los experimentadores, y rural, para consumidores actuales. No se observó una modificación del efecto según intoxicación aguda por alcohol. Sin embargo, los hombres con bajo desempeño escolar incrementaron la posibilidad de experimentar con tabaco, si tenían el antecedente de consumo de drogas ( $R M=2.9$ IC 95\%:1.4-6.0) de manera más marcada en los fumadores actuales ( $R M=4.5$ IC 95\%:2.1-9.5). Se aprecia un gradiente dosis respuesta significativo entre bajo desempeño escolar y el antecedente de tabaquismo en el padre $(p<0.05)$, pero no en el caso de que la madre sea fumadora.

\section{Discusión}

Los resultados del estudio del estado de Morelos en México brindan evidencia empírica de la relación li- 

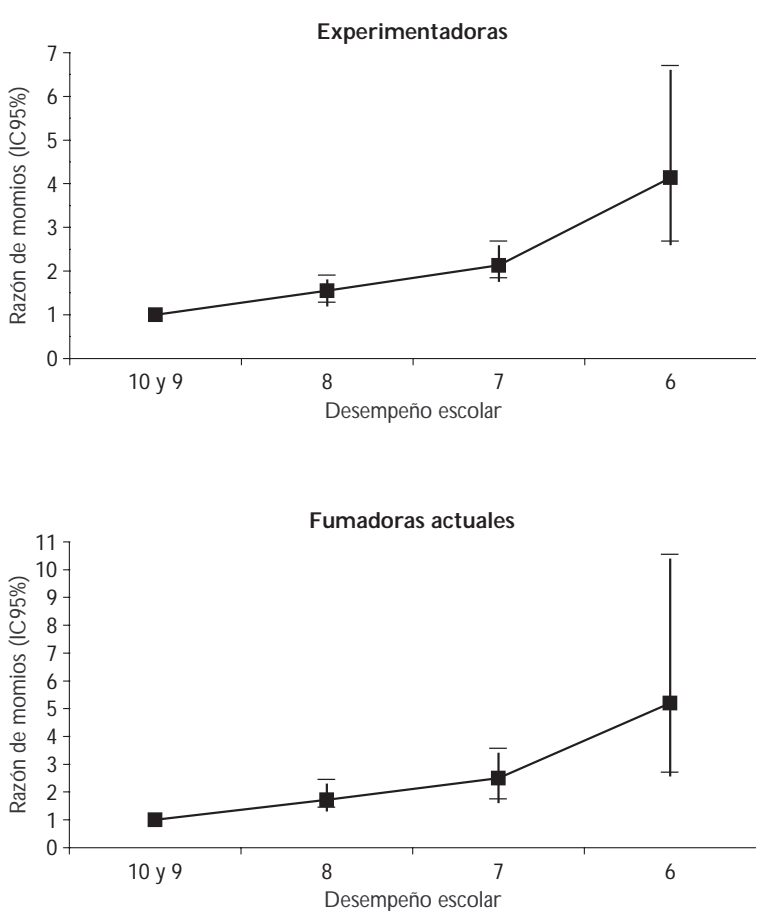

Figura 1. Desempeño escolar y hábito tabáquico en las estudiantes del estado de Morelos, México, 1998-1999

neal entre bajo desempeño escolar y el incremento de consumo de tabaco, independientemente del efecto de otras variables de exposición estudiadas; asociación estrechamente observada en ambos géneros.

Diversos autores coinciden en explicar la asociación entre comportamientos de riesgo -tales como consumo de tabaco, alcohol, drogas ilícitas, pobre desempeño escolar- como parte de ciertos estilos de vida, ${ }^{24}$ mismos que se definen como patrones colectivos de comportamiento de salud relacionados con alternativas provenientes de opciones disponibles del sujeto de acuerdo con sus oportunidades de vida. Según Cockerham, esta definición incorpora la contribución central de Max Weber a la teoría de estilo de vida, que establece que las decisiones y las oportunidades de vida interactúan unas con otras. Aunque él pone énfasis en el rol de las alternativas, no pasa por alto que las oportunidades de la vida son una condición estructural, es decir, dependen de su posición socioeconómica en la sociedad. ${ }^{25,26}$

Los estilos de vida, según Pierre Bourdieu, son las prácticas cotidianas de un sujeto, que reflejan los habi-
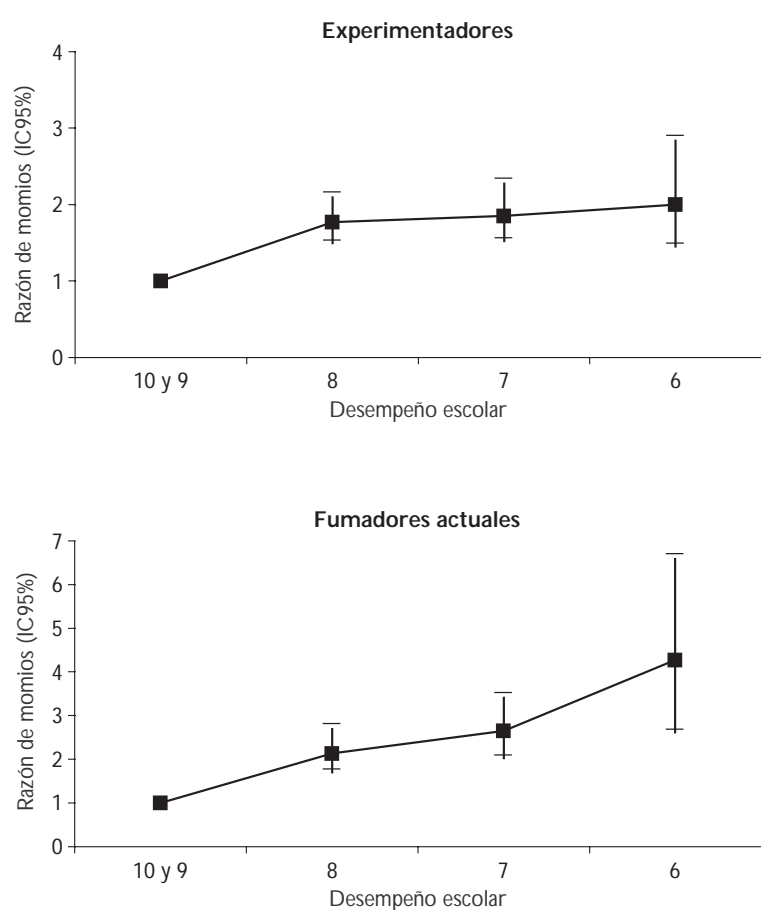

Figura 2. Desempeño escolar y hábito tabáquico en los estudiantes del estado de Morelos, México, 1998-1999

tus* basados en las experiencias, socialización y la realidad de clase social. ${ }^{27} \mathrm{El}$ habitus está constituido por todas estas disposiciones inconscientes que guían y marcan las pautas por ciertos comportamientos, que se expresan en estilos de vida.

Conviene mencionar que la cultura, los ingresos, la estructura familiar, la edad, la capacidad física, el entorno doméstico y laboral, harán más atractivas, factibles y adecuadas determinadas formas y condiciones de vida. En este contexto, las condiciones de vida son el entorno cotidiano de las personas, donde éstas viven, actúan y trabajan. Son, asimismo, el producto de circunstancias sociales, económicas y del entorno

* Pierre Bourdieu define al habitus "como un sistema de disposiciones durables y transferibles -estructuras estructuradas predispuestas a funcionar como estructuras estructurantes- que integran todas las experiencias pasadas y funciona en cada momento como matriz estructurante de las percepciones, las apreciaciones y las acciones de los agentes cara a una coyuntura o acontecimiento y que él contribuye a producir". 


\section{Cuadro V \\ AsOCIACIÓN* ENTRE EL DESEMPEÑO ESCOLAR Y CONSUMO DE TABACO EN LOS ESTUDIANTES, SEGÚN DIFERENTES VARIABLES SOCIECONÓMICAS, ANTECEDENTES PERSONALES Y FAMILIARES. Morelos, México, 1998-1999}

\begin{tabular}{|c|c|c|c|c|c|c|c|c|c|}
\hline \multirow[b]{2}{*}{$\begin{array}{l}\text { Desempeño } \\
\text { escolar }\end{array}$} & \multirow[b]{2}{*}{$10-9^{\ddagger}$} & \multicolumn{4}{|c|}{ Experimentadores } & \multicolumn{4}{|c|}{ Fumadores actuales } \\
\hline & & $\begin{array}{c}8 \\
\text { RM IC } 95 \%\end{array}$ & $\begin{array}{c}7 \\
\text { RM IC } 95 \%\end{array}$ & $\begin{array}{c}\leq 6 \\
\text { RM IC } 95 \%\end{array}$ & $\begin{array}{l}\chi^{2} \text { tendencia } \\
\text { (p) }\end{array}$ & $\begin{array}{c}8 \\
\text { RM IC } 95 \%\end{array}$ & $\begin{array}{c}7 \\
\text { RM IC } 95 \%\end{array}$ & RM IC 95\% & $\begin{array}{l}\chi^{2} \text { tendencia } \\
\text { (p) }\end{array}$ \\
\hline Modelo total & 1.0 & $1.8(1.5-2.1)$ & $1.9(1.6-2.3)$ & $2.0(1.4-2.9)$ & $41.4(p<0.001)$ & $2.1(1.6-2.8)$ & $2.6(2.0-3.5)$ & $4.2(2.7-6.7)$ & $48.8(p<0.001)$ \\
\hline \multicolumn{10}{|l|}{ Edad (en años) } \\
\hline $11-12$ & 1.0 & $1.2(1.0-1.7)$ & $1.2(0.9-1.8)$ & $1.4(0.6-3.0)$ & $1.8(p=0.18)$ & $1.7(1.1-2.9)$ & $2.5(1.5-4.3)$ & $6.1(2.4-15.1)$ & $17.0(p<0.001)$ \\
\hline $13-14$ & 1.0 & $1.9(1.5-2.4)$ & $2.3(1.7-3.0)$ & $2.6(1.6-4.3)$ & $34.0(p<0.001)$ & $2.2(1.4-3.4)$ & $2.4(1.5-3.8)$ & $3.1(1.4-6.8)$ & $10.4(p<0.01)$ \\
\hline $15-17$ & 1.0 & $2.4(1.5-3.9)$ & $2.4(1.4-4.0)$ & $2.2(0.9-5.3)$ & $8.4(p<0.01)$ & $2.5(1.3-4.9)$ & $4.1(2.0-8.2)$ & $3.7(1.2-11.0)$ & $13.6(p<0.001)$ \\
\hline $18-24$ & 1.0 & $2.6(1.4-4.7)$ & $2.4(1.3-4.6)$ & $2.3(0.6-8.8)$ & $6.0(p<0.05)$ & $2.5(1.3-4.7)$ & $2.3(1.2-4.6)$ & $5.1(1.5-17.1)$ & $6.6(p<0.05)$ \\
\hline
\end{tabular}

Indice de nivel socioeconómico

\begin{tabular}{llllllllll} 
Bajo & 1.0 & $1.7(1.1-2.5)$ & $1.3(0.8-2.0)$ & $1.3(0.6-2.8)$ & $0.4(p=0.52)$ & $2.8(1.4-5.7)$ & $2.5(1.2-5.2)$ & $2.9(1.0-8.7)$ & $3.4(p<0.06)$ \\
\hline Medio & 1.0 & $1.7(1.4-2.1)$ & $2.0(1.6-2.6)$ & $2.5(1.6-3.9)$ & $36.2(p<0.001)$ & $2.3(1.5-3.5)$ & $3.0(1.9-4.7)$ & $6.0(3.1-11.5)$ & $29.2(p<0.001)$ \\
\hline Alto & 1.0 & $2.1(1.5-3.1)$ & $2.4(1.6-3.7)$ & $1.7(0.5-5.1)$ & $13.6(p<0.001)$ & $1.9(1.3-2.9)$ & $2.6(1.7-4.1)$ & $2.7(1.0-7.0)$ & $16.4(p<0.001)$
\end{tabular}

Lugar de residencia

\begin{tabular}{lllllllllc} 
Rural & 1.0 & $1.6(1.2-2.1)$ & $1.9(1.4-2.6)$ & $1.8(1.1-3.1)$ & $17.8(p<0.001)$ & $2.0(1.2-3.6)$ & $2.5(1.4-4.6)$ & $5.0(2.3-11.2)$ & $11.8(p<0.001)$ \\
\hline Semiurbana & 1.0 & $1.7(1.2-2.3)$ & $1.7(1.1-2.4)$ & $2.0(0.9-4.3)$ & $7.0(p<0.01)$ & $3.7(1.9-7.4)$ & $3.2(1.5-6.6)$ & $4.6(1.5-14.5)$ & $6.4(p<0.05)$ \\
\hline Urbana & 1.0 & $2.1(1.6-2.8)$ & $2.1(1.5-2.9)$ & $2.4(1.2-4.7)$ & $17.7(p<0.001)$ & $2.0(1.4-2.8)$ & $2.7(1.8-3.9)$ & $3.5(1.7-7.2)$ & $27.0(p<0.001)$
\end{tabular}

Intoxicación aguda por alcohol

\begin{tabular}{cccccccc} 
No & 1.0 & $1.7(1.5-2.1)$ & $1.9(1.6-2.3)^{\S}$ & $44.4(p<0.001)$ & $2.1(1.6-2.8)$ & $2.8(2.1-3.8)^{\S}$ & $46.0(p<0.001)$ \\
\hline Sí & 1.0 & $2.2(0.8-5.8)$ & $1.6(0.6-4.3)^{\S}$ & $0.6(p=0.43)$ & $2.4(0.9-6.3)$ & $2.7(1.0-7.4)^{\S}$ & $2.2(p=0.13)$
\end{tabular}

Consumo de alguna droga

\begin{tabular}{llllllll} 
N 0 & 1.0 & $1.7(1.4-2.0)$ & $1.9(1.5-2.3)^{\S}$ & $37.6(p<0.001)$ & $2.1(1.6-2.8)$ & $2.7(2.0-3.7)^{\S}$ & $37.0(p<0.001)$ \\
\hline Sí & 1.0 & $2.8(1.4-5.6)$ & $2.9(1.4-6.0)^{\S}$ & $6.8(p<0.01)$ & $3.3(1.6-6.8)$ & $4.5(2.1-9.5)^{\S}$ & $12.2(p<0.001)$
\end{tabular}

Hábito tabáquico del padre

\begin{tabular}{cccccccc} 
N 0 & 1.0 & $1.8(1.5-2.2)$ & $2.0(1.6-2.4)^{\S}$ & $40.0(p<0.001)$ & $2.4(1.7-3.3)$ & $3.2(2.3-4.4)^{\S}$ & $43.4(p<0.001)$ \\
\hline Sí & 1.0 & $1.6(1.1-2.4)$ & $1.6(1.1-2.5)^{\S}$ & $4.4(p<0.05)$ & $1.5(0.9-2.5)$ & $2.0(1.2-3.5)^{\S}$ & $6.2(p<0.05)$
\end{tabular}

Hábito tabáquico de la madre

\begin{tabular}{lrrrrrrr} 
No & 1.0 & $1.8(1.5-2.1)$ & $2.0(1.6-2.4)^{\S}$ & $45.8(p<0.001)$ & $2.1(1.6-2.8)$ & $3.0(2.2-4.0)^{5}$ & $50.2(p<0.001)$ \\
\hline Sí & 1.0 & $1.5(0.7-3.2)$ & $1.1(0.5-2.4)^{\S}$ & $0.02(p=0.88)$ & $2.4(1.0-5.7)$ & $1.8(0.7-4.6)^{\S}$ & $0.5(p=0.49)$
\end{tabular}

* RM ajustada por edad, nivel socioeconómico, lugar de residencia, intoxicación aguda por consumo de alcohol al menos una vez al mes, consumo pasado 0 actual de alguna droga ilegal, hábito tabáquico del padre y de la madre

₹ Categoría de referencia

$\S$ RM para puntuación en el desempeño escolar $\leq 7$ 
físico, lo cual ejerce un impacto en la salud, estando en gran medida fuera del control inmediato del individuo. ${ }^{25}$

Como lo menciona Menéndez, ${ }^{28}$ en algunos casos, la aplicación biomédica "extrajo" el padecimiento de los procesos culturales y sociales en que se desarrolla, y utilizó el concepto de estilo de vida para describir rasgos específicos de comportamientos relacionados en un principio con enfermedades crónicas, y más tarde con adicciones y violencias. Implícitamente podría estar la noción de que el propio sujeto puede elegir y actuar, intencional y responsablemente, pero existe una influencia social sobre él aunque no lo reconozca, empezando por su propia condición socioeconómica. En este contexto, la reducción o eliminación del hábito tabáquico no puede ser explicada exclusivamente a partir del comportamiento específico e individual. ${ }^{28} \mathrm{En}$ general, la gente socialmente marginada tiene menos autonomía para elegir comportamientos saludables; por ejemplo, el acceso a recursos e información en salud es más restringido. ${ }^{24}$ Menéndez propone que el estilo de vida debe ser abordado con una visión holística, reconocer que dentro de cada grupo, estrato o clase social, existen variables en el sujeto y microgrupo. ${ }^{28}$

Los estilos de vida, en resumen, son una actividad colectiva que va más allá de la psicología individual de las personas, y que está sustentada en las normas, valores y en las prácticas de grupos, clases sociales y sociedad que configuran y establecen parámetros para el comportamiento individual cotidiano. ${ }^{25}$ Así, cuando se intenta explicar la asociación entre desempeño escolar y consumo de tabaco, dicha relación puede ser abordada dentro del marco de estilos de vida.

Como ya se mencionó, este estudio muestra una asociación inversa entre el autorreporte del desempeño escolar y el consumo de tabaco en estudiantes de Morelos. Resultados similares a los de este trabajo, han sido evidenciados por estudios en otras poblaciones que han analizado la autopercepción del desempeño escolar, encontrando que estudiantes fumadores presentan una mayor probabilidad de tener un pobre desempeño escolar, en comparación con los no fumadores. ${ }^{13}$ Otros estudios han usado evaluaciones específicas para valorar el desempeño escolar con hallazgos similares; así también con asociación a consumo de alcohol y otras drogas, en ambos géneros. ${ }^{18,20}$ En contraposición, un estudio en Indonesia mostró que variables relacionadas con la escuela (gusto por la escuela, llegar tarde a clases, presión por tareas escolares y desempeño escolar) jugaron un rol menos importante del que se esperaba. Los propios autores mencionan como probable explicación que esta socie- dad tiende a evitar los conflictos, influyendo así la orientación de las respuestas en lo que es socialmente deseable. $^{14}$

Tyas y Pederson, ${ }^{10}$ en su trabajo de revisión de la literatura, reportan que hay una consistencia de la asociación inversa entre consumo de tabaco y desempeño escolar, lo que también ha sido asociado con aspiraciones escolares y compromiso con la escuela. Así, aquellos estudiantes con un buen desempeño escolar, que tienen altas aspiraciones académicas y están comprometidos con su educación, presentan una menor probabilidad de fumar que aquellos que no poseen estas características. Para estas autoras, el efecto protector de estas características puede reflejar creencias necesarias para el éxito académico.

El éxito escolar incluye componentes de motivación, expectativas y compromiso educativo, así como sentido de control sobre el presente y el futuro. ${ }^{11}$ Asimismo, existen otros determinantes del desempeño escolar, aquellos intrínsecos al sujeto (coeficiente intelectual, aspectos psicológicos, motivación, creencias, hábitos, estado nutricio etcétera), así como del ámbito sociofamiliar (ambiente cultural familiar, tipo de familia, grupo de amigos y compañeros, etcétera), y otros propios de la institución educativa (enfoque pedagógico, curriculum escolar, características de la escuela, capacidad y expectativas de los profesores y metodología didáctica), por mencionar algunos. ${ }^{16} \mathrm{In}$ dependiente del consumo de tabaco, los estudiantes con menor desempeño escolar parecen tener conductas destructivas y menor apego a normas, ${ }^{29}$ o mayor agresividad. ${ }^{16}$

Trabajos previos han intentado establecer si el consumo de tabaco, alcohol y drogas ilícitas está determinado por un bajo desempeño escolar, o esta relación es inversa. ${ }^{11,19}$ Por una parte, la evidencia indica que el compromiso y éxito educativo en la escuela están inversamente relacionados con uso de sustancias y con otros problemas anteriores de comportamiento. Dentro de la literatura sobre delincuencia, se señala el bajo éxito educativo como un precursor de riesgo para los actos delictivos, incluyendo el uso de sustancias ilegales. ${ }^{30}$ Asimismo, en las teorías de la frustración, la falta de éxito educativo lleva a la percepción de carencia de oportunidades futuras, ${ }^{11}$ lo cual, en muchas ocasiones, hace emerger el uso de drogas ilícitas y la realización de actos delictivos, como respuesta compensatoria a la frustración de un desempeño escolar pobre. ${ }^{31}$

En contraparte, otras evidencias indican que fumar puede conducir al abuso de drogas y a conductas delictivas; a su vez, el conjunto de ellas tiene un efecto negativo en el desempeño escolar. ${ }^{11}$ Así también, el uso 
temprano de sustancias puede contribuir a dificultades escolares y hasta inducir la deserción escolar, sugiriendo que las adicciones propician insatisfacción por la escuela, actitudes negativas, decremento de la motivación e incremento en la tasa de ausentismo. ${ }^{32,33}$

En este contexto, sin embargo, es posible que el funcionamiento escolar, el psicosocial y el uso de sustancias se influyan unos a otros de una manera recíproca a través del tiempo, especialmente en los adolescentes más jóvenes, ${ }^{19}$ debido a las características intrínsecas de la etapa de la adolescencia.

Respecto de la variable edad, es evidente el típico incremento de la prevalencia de consumo de tabaco, conforme la edad avanza, reportado en otros estudios; $;^{10,11,13,34}$ aunque el grupo de 11 a 12 doce muestra una fuerte asociación en ambos géneros en fumadores actuales.

El patrón diferencial que se observa en el comportamiento de mujeres y hombres ha sido parcialmente descrito por Flay. ${ }^{20}$ Como se observa en los resultados, en el grupo estudiado la prevalencia es menor en las mujeres que en hombres, como se muestra en general en otros estudios, ${ }^{13,15}$ aunque se ha dado una progresiva aceptación social del consumo de tabaco por parte de la población femenina, como símbolo de la emancipación de las mujeres y de la liberación de los roles tradicionales de género, lo que ha contribuido a su incremento., ${ }^{92,35}$ Además, según el modelo de López ${ }^{6}$ y otros estudios de tendencias internacionales, $, 36,37$ es de esperar un incremento de consumo de tabaco en la población femenina. En los EUA se ha reportado que no existen diferencias en la prevalencia de consumo entre mujeres y hombres estudiantes; ${ }^{11,18}$ en países como el Reino Unido, el consumo de tabaco en estudiantes es ya más común en mujeres que en hombres.

En los resultados de este trabajo, los estudiantes de uno u otro sexo, con el más alto estrato socioeconómico en la muestra estudiada, tienen una mayor prevalencia de consumo de tabaco, lo cual es similar, según otros estudios, en otras sociedades. ${ }^{38}$

Respecto del lugar de residencia, en el caso de las estudiantes de Morelos existe una mayor prevalencia de tabaquismo en las del área semiurbana y en varones del área rural. A este respecto, con base en el modelo teórico de López, ${ }^{6}$ la epidemia de tabaquismo inicia con un incremento en áreas urbanas, por estar más expuestas a la publicidad, entre otras condiciones, y posteriormente en áreas semiurbanas y rurales; respecto al género, el hábito tabáquico es adquirido primero por los hombres, por lo que nuestros hallazgos son acordes con dicho modelo.

En este estudio, la intoxicación aguda por alcohol al menos una vez al mes presenta una fuerte asocia- ción independiente con la experimentación y con el consumo actual de tabaco, tal y como ha sido ampliamente descrito. ${ }^{10,15,17,18,20}$ En las mujeres y, en menor medida en los hombres, que han presentado algún episodio de intoxicación aguda, la asociación con el desempeño escolar es menor; mientras que en los estudiantes que no han presentado intoxicación aguda por alcohol se mantiene la relación inversa entre desempeño escolar y consumo de tabaco. Esto puede deberse a la mayor influencia de la intoxicación aguda sobre el comportamiento de experimentación o consumo de tabaco actual, que hace perder casi toda la influencia de la variable desempeño escolar. No se puede excluir que la baja prevalencia de intoxicación aguda por alcohol pueda haber afectado la estimación estadística de los datos. Un mecanismo similar podría explicar la relación con el consumo de alguna droga ilegal, la cual mostró una fuerte asociación con el hábito tabáquico, en éste y otros estudios. ${ }^{10,15,16-18}$

En cuanto a la percepción de los estudiantes sobre el antecedente de tabaquismo en los padres, trabajos previos reportaron que puede tener más impacto en las mujeres que en los hombres. ${ }^{10,20}$ En el presente estudio, las mujeres experimentadoras muestran su consumo asociado con el tabaquismo de ambos padres, asociación que no es tan clara en fumadoras actuales, como ya había sido también reportado en la literatura. ${ }^{10}$ En el caso de los hombres, sólo es aparente la asociación cuando el padre es fumador, y no así cuando lo es la madre, como lo muestran otros trabajos. ${ }^{10,20}$ Esta relación la explica Wickrama ${ }^{24}$ a partir de una transmisión intergeneracional de los estilos de vida, comportamientos de salud y riesgo, de padres a hijos.

La relación inversa entre consumo de tabaco y desempeño escolar es claramente evidente cuando los padres no fuman, pero en presencia del antecedente de tabaquismo en los padres esta relación desaparece porque es más fuerte la asociación con esta variable; fenómeno similar al ya observado para el caso del antecedente de intoxicación aguda por alcohol.

Una de las potenciales limitaciones de este trabajo es inherente al tipo de diseño, pues al tratarse de un estudio transversal, los efectos encontrados sólo pueden ser interpretados como asociaciones entre las variables de estudio y no como relaciones causales. Respecto del posible sesgo de clasificación de los fumadores, al basarse en un autorreporte de los encuestados, podría subestimar la cantidad o la frecuencia, ${ }^{39}$ aunque la declaración del hábito se considera una estimación válida de la conducta real, ${ }^{40}$ incluso en adolescentes. ${ }^{41}$ Respecto de los sesgos de selección, se tendría que considerar si existe alguna característica diferente en los estudiantes que no contestaron la 
encuesta y los que sí: una posibilidad podría ser que existiera ausentismo por problemas de salud ocasionados ya por el consumo de tabaco. Sin embargo, la tasa de no respuesta fue sólo de $1.4 \%$, con lo que cualquier sesgo debido a la no participación queda prácticamente descartado.

Llama la atención que las mujeres informen un consumo de tabaco menor de sus padres, en comparación con los hombres. Una posible explicación es que los valores y normas transmitidos a las mujeres están más orientados a los comportamientos que la sociedad acepta como deseables. ${ }^{35}$ La Encuesta Nacional sobre Consumo de Drogas en la Comunidad Escolar de la Ciudad de México en 1997, ${ }^{42}$ halló que la tolerancia social al tabaco percibida por las mujeres es más baja que la percibida por los hombres. Así también, en estudiantes suecas se encontró que están más comprometidas con mejores comportamientos saludables que sus compañeros hombres. ${ }^{43}$ En este estudio probablemente se esté subestimando la prevalencia de consumo de tabaco en los padres por el autorreporte de las estudiantes. Sin embargo, los factores asociados con consumo de tabaco en la población de estudio fueron consistentemente similares en la mayor parte de las variables en ambos géneros.

Los potenciales factores de confusión se han controlado mediante la inclusión de las variables relacionadas con el hábito tabáquico en cada uno de los modelos. La asociación entre desempeño escolar y el hábito tabáquico persiste al ajustar por el resto de variables, que también mantuvieron una asociación independiente con el hábito (excepto el índice de nivel socioeconómico y el área de residencia). Además, se han ajustado los modelos según diferentes estratos del resto de variables para comprobar si la asociación se modificaba. En este sentido, el patrón de asociación inversa se ha atenuado en algunos estratos, debido a la fuerte asociación entre tabaco y determinadas variables (intoxicación aguda por alcohol y antecedente de tabaquismo en los padres).

Los hallazgos de este estudio aportan elementos para confirmar una relación inversa entre consumo de tabaco y bajo rendimiento escolar, que pueden ser indicadores de un cierto estilo de vida del estudiante, interrelacionados con otros comportamientos de riesgo, como consumo de alcohol y drogas ilegales. Son necesarios posteriores estudios prospectivos de seguimiento para profundizar en dicha asociación y caracterizar las variables que condicionan la instauración definitiva y el mantenimiento del hábito tabáquico. Cabe mencionar que se ha reportado que el bajo desempeño escolar en estudiantes fumadores está aso- ciado también con una menor probabilidad de abandono del hábito tabáquico. ${ }^{11}$

Es necesario llamar la atención sobre otros efectos nocivos del consumo de tabaco, alcohol y drogas ilegales, que son potenciales precursores de la deserción escolar, ${ }^{33,44}$ con las conocidas implicaciones sociales para los adolescentes y adultos jóvenes. ${ }^{45}$

Finalmente, la identificación de conductas de riesgo asociadas diferencialmente con el tabaquismo en etapa temprana, puede ser de ayuda para establecer intervenciones educativas que desalienten su consumo y modifiquen las pautas de conducta ya establecidas. En este contexto, las propuestas de intervenciones deben plantearse en forma integral, focalizadas en ciertos estilos de vida de los grupos de interés. Además, es fundamental incorporar una perspectiva de género que considere las diferencias en roles, valores, percepciones, y características personales entre el hombre y la mujer.

\section{Agradecimientos}

Se agradecen los comentarios de las doctoras Adriana Ramírez, Stella Araujo y del doctor Raúl Sansores, así como la asesoría técnica de Anna Schiaffino en el análisis de los datos. Las Secretarías de Educación Pública y de Salud, del estado de Morelos, brindaron todas las facilidades para realizar el estudio.

\section{Referencias}

1. Peto R, López AD, Boreham J,Thun M, Heath C, D oll R. Mortality from smoking worldwide. Br Med Bull 1996;52:12-21.

2. World Health Organization. The W orld Health Report 1995. Bridging the gaps. Ginebra:W orld Health O rganization, 1995.

3. Secretaría de Salud. Encuesta N acional de Adicciones 1998. México, D.F.: SSA, 1999.

4. Secretaría de Salud. Encuesta Nacional de Adicciones 1988. México, D.F.: SSA, 1990.

5. Medina-Mora ME, De La Fuente R. Estudio nacional sobre uso de drogas en la población estudiantil de la República Mexicana. En: Rubio $\mathrm{H}$, Labrandero M, Selman M, Martínez D ossier J, Pérez N eria J,Yánez RM et al. Avances del Programa contra el Tabaquismo.

6. López A, Collishaw N, PihaT.A descriptive model of the cigarette epidemic in developed countries. Tobacco Control 1994;3:242-247.

7. Lazcano EC, Tovar V, Meneses F, Rascón RA, Hernández M.Trends of lung cancer mortality in Mexico.Arch Med Res 1997;28:565-570.

8. Roth L, Taylor H. Risks of smoking to reproductive health: Assessment of women's knowledge. Am J O bstet Gynecol 2001;184:934-939.

9. Ernster V, Kaufman N , N ichter M, Samet J,Yoon SY. Las mujeres y el tabaco: de la política a la acción. Bol 0 rgan Mundial Salud 2001;78: 891-901.

10.Tyas S, Pederson L. Psychosocial factors related to adolescent smoking: A critical review of the literature.Tobacco Control 1998;7:409-420. 
11. HuTw, Lin Z, Keeler TE.Teenage smoking, attempts to quit, and school performance. Am J Public Health 1998;88:940-943.

12. Stein KF, Roeser R, Markus HR. Self-schemas and possible selves as predictors and outcomes of risky behaviors in adolescents. Nurs Res 1998:47:96-106.

13. Flay BR, HU FB, Richardson J. Psychosocial predictors of different stages of cigarette smoking among high school students. Prev Med 1998;27: A9-A18.

14. Samet B, Maes L, De Clercq L, Haryanti K, D jati R. Determinants of smoking behavior among adolescents in Semarang, Indonesia. Tobacco Control 1999:8:186-191.

15. Azevedo A, Machado AP, Barros H. Tobacco smoking among Portuguese high-school students. Bull W orld Health O rgan 1999;77:509-514.

16. W alker S, G rantham-McG regor S, Himes J, W illiams S, D uff E. School performance in adolescent Jamaica girls: Associations with health, social and behavioral characteristics, and risk factors for dropout,J A dolesc 1998; 21:109-122.

17. Miller PM, Plant M. D rinking, smoking, and illicit drug use among 15 and 16 years olds in the United Kingdom. BMJ 1996;17(313):394-397.

18. Dappen A, Schwartz RH, O 'donnell R.A survey of adolescent smoking patterns. J Am Board Fam Pract 1996;9:7-13.

19. Schulenberg J, Bachman J, 0 'Malley P, Johnston L. High school educational success and subsequent substance use: A panel analysis following adolescents into young adulthood.J Health Soc Behav 1994;35:45-62.

20. Flay BR, Hu FB, Richardson J. Psychosocial predictors of different stages of cigarette smoking among high school students. Prev Med 1998;27: A9-A18.

21.Amos A.W omen and smoking. Br Med Bull 1996;52:74-89.

22. Joossens L, Saco A, Salvador T, Villalbí J. Las mujeres y el tabaco en la Unión Europea. Rev Esp Salud Publica 1999;73:3-11.

23. Kleinbaum D. Una introducción al análisis de regresión logística. En: Porta M,Alvarez-D ardet C, ed. Rev Salud Publica 1993;3:61-105.

24.W ickkama $\mathrm{K}$, C onder R,W allace L, Elder $\mathrm{G}$. The intergeneration transmission of health-risk behaviors: Adolescent lifestyles and gender moderating effects. J Health Soc Behav 1999;40:258-272.

25. 0 rganización Mundial de la Salud. G losario de términos. Ginebra:W HO, 1998.

26. Cockerham W. Health lifestyles in Russia. Soc Sci Med 2000;51: 1313-1324.

27. Bourdieu P. La distinción. C riterios y bases sociales del gusto. Madrid: Editorial Taurus, 1988;169-222.

28. Menéndez E. Estilos de vida, riesgos y construcción social. Conceptos similares y significados diferentes. Est Sociol XVI 1998;46:37-67.
29. Caldas S. Multilevel examination of student, school, and district-level effects on academic achievement.J Educ Res 1999;93:91-101.

30. Hirschi, Travis. Causes of delinquency. Berkeley: U niversity of $C$ alifornia Press, 1969.

31. Leiber M, Farnsworth M. Strain theory revised: Economic goals, educational means, and delinquency. Am Sociol Rev 1989;54:263-274.

32. Mensch B, Kandel D. Dropping out of high school and drug involvement. Sociol Educ 1988;61:95-113.

33. Lynskey M, Hal W. The effects of adolescent cannabis use on educational attainment:A review. Addiction 2000;95:1621-1630.

34. W ang SQ , Yu JJ, Z hu BP, et al. Cigarette smoking and its factors among senior high school students in Beijing, China, 1988. Tobacco Control 1994;3:107-114.

35. W aldrom I. Patterns and causes of gender differences in smoking. Soc Sci Med 1991;32:989-1005

36. Molarius A, et al. Trends in cigarette smoking in 36 populations from the early 1980s to the mid-1990s: Findings from the W HO MONICA Project.Am J Public Health 2001;91:206-212.

37. Fernández E, Schiaffino A, Borrás JM. Epidemiología del tabaquismo en Europa. Salud Publica Mex; 2001.

38. Fernández E, García M, Schiaffino A, Borrás JM, N ebot M, Segura A. Smoking initiation and cessation by gender and educational level in $\mathrm{C}$ ataIonia, Spain. Prev Med 2001;32.

39. Patrick D L, C headle A, Thompson DC , Diehr P, Koepsell T, Kinne S.The validity of self-reported smoking:A review and meta-analysis. Am J Public Health 1994;84(7):1086-1093.

40. MorabiaA, Bernstein M, C urtin F, Berode M.Validation of self-reported smoking status by simultaneous measurement of carbon monoxide and salivary thiyanate. Prev Med 2001;32:82-88.

41. Dolcini MM,Adler N E, Ginsberg D. Factors influencing agreement between self-reports and biological measures of smoking among adolescents. J Res Adolesc 1996;6:515-542.

42. Secretaría de Educación Pública. Instituto Mexicano de Psiquiatría. Encuesta N acional sobre Consumo de Drogas entre la Comunidad Escolar. México, D.F.: SEP, IMP, 1997

43. N aslund GK, Fredrikson M. Health behavior, knowledge and attitudes among Swedish university students. Scand J Psychol 1993;34:197-211.

44. Bray J, Zarkin G, Ringwalt C, Q i J.The relationship between marijuana initiation and dropping out of high school. Health Econ 2000;9:9-18.

45. Kawachi I. Income inequality and health. En: Berkman L, Kawachi I, comp. Social epidemiology. 0 xford, USA: 0 xford University Press; 2000: 76-94. 